\title{
Real-Time Cardiac Arrhythmia Detection Using WOLA Filterbank Analysis of EGM Signals
}

\author{
Hamid Sheikhzadeh, Robert L. Brennan, and Simon So \\ AMI Semiconductor Canada Company, 611 Kumpf Drive, Unit 200, Waterloo, Ontario, Canada N2V 1 K8
}

Received 27 April 2006; Revised 13 October 2006; Accepted 13 October 2006

Recommended by William Allan Sandham

Novel methods of cardiac rhythm detection are proposed that are based on time-frequency analysis by a weighted overlap-add (WOLA) oversampled filterbank. Cardiac signals are obtained from intracardiac electrograms and decomposed into the timefrequency domain and analyzed by parallel peak detectors in selected frequency subbands. The coherence (synchrony) of the subband peaks is analyzed and employed to detect an optimal peak sequence representing the beat locations. By further analysis of the synchrony of the subband beats and the periodicity and regularity of the optimal beat, various possible cardiac events (including fibrillation, flutter, and tachycardia) are detected. The Ann Arbor Electrogram Library is used to evaluate the proposed detection method in clean and in additive noise. The evaluation results show that the method never misses any episode of fibrillation or flutter in clean or in noise and is robust to far-field R-wave interference. Furthermore, all other misclassification errors were within the acceptable limits.

Copyright (c) 2007 Hamid Sheikhzadeh et al. This is an open access article distributed under the Creative Commons Attribution License, which permits unrestricted use, distribution, and reproduction in any medium, provided the original work is properly cited.

\section{INTRODUCTION}

The objective of this research is rhythm classification and event detection based on the intracardiac electrogram (EGM) signals. The proposed methods are designed for implantable devices that should operate on extremely lowpower budgets. In the meantime, these methods should operate in real time and the processing delay should be in the minimal range acceptable for such applications. The detection methods should be very reliable and robust to interference, noise, and morphology variations.

Current practical methods of cardiac rhythm detection employed in implantable cardioverter defibrillators (ICDs) are generally based on beat-by-beat time-domain analysis. Although research has been conducted to exploit more sophisticated signal processing such as wavelet transform and template matching for event detection [1-3], the new methods have rarely been employed in practical systems due to their computational and power demands and issues related to the reliability of their detection.

Current challenges in reliable rhythm detection for implantable cardiac rhythm management (CRM) systems such as ICDs are the following.
(1) Inappropriate device therapy (IDT) amount to a considerable rate (between 10 to $30 \%$ ) in various devices [4]. IDTs occur due to low EGM signal quality, sinus tachycardia, supraventricular tachycardia (SVT), myopotential interference, external interference, and Twave oversensing [5]. IDT is painful to the patient and depletes the device battery power more quickly. IDT is also potentially harmful to the patient as it puts the patient at risk of device-induced VT (proarrhythmia) that might be dangerous and hard to detect by the ICD $[4,5]$.

(2) Missing serious cardiac events compromises the reliability of the CRM devices. This happens due to many reasons including quick morphology, rate, and even polarity changes of the EGM signal, abnormally wide $\mathrm{R}$-waves and $\mathrm{P}$-waves, and external noises [1]. The problems are aggravated due to the fact that often patients have to simultaneously use medicines that alter the EGM waveforms.

(3) Following a device therapy (low-energy pacing or high-energy cardioversion), it is essential to quickly redetect the EGM rhythm to analyze the effectiveness of the therapy. Quick redetection, however, is tricky as 
often the device therapy polarizes the EGM electrodes or causes baseline variations of the EGM signal (sometimes in form of low-frequency oscillations with magnitudes larger than the EGM beat itself). Often a blackout period has to be applied before any reliable new sensing.

(4) As increasingly sophisticated multichamber CRM devices become available offering the physicians more choices in programming the devices, more powerful signal processing is required to efficiently handle the multichannel information while offering the physician less complicated and reliable programming scenarios. Also, increased immunity to cross-channel interference is necessary.

To cope with the mentioned problems and to improve the rhythm detection accuracy, we propose a multitiered detection method that is based on time-frequency analysis of the EGM signals by a weighted overlap-add (WOLA) filterbank. The WOLA filterbank can be efficiently implemented on an ultra-low power platform [6] targeted for real-time low-delay and implantable devices.

Methods based on time-frequency analysis have already been proposed for electrocardiogram (ECG) and QRS detection [7]. However, the proposed methods are not qualified for ultra-low power implementation on implantable devices since they are either (a) too complex or unsuitable for realtime applications or (b) specifically designed for (and evaluated on) ECG signals and do not provide the robust and reliable performance essential for EGM signal processing.

Possibly, the most relevant to our work is the research by Afonso et al. on ECG beat detection in real time [8]. They analyze the ECG signal by a critically sampled polyphase filterbank, extracting six features that are all based on the accumulated energy (or absolute value) of groups of subbands. Each energy feature is processed by a peak detector that compares the signal moving average to a threshold that is determined based on an estimation of the background noise. Peaks are then refined in a cascade of five stages (levels). In one stage, two different thresholds are used to detect the peaks of the same feature. The peaks are then combined in parallel. The method is evaluated on a standard ECG database with satisfactory performance.

The objective of this research is to present a real-time detection method for cardiac event detection using the intracardiac EGM signals. Sensed EGM signals differ from ECG signals in many aspects. Major differences are summarized here.

(1) EGM signals provide direct access to individual heart chambers, most importantly right ventricular and right atrial, at the signal source. In contrast, ECG signals provide a combined signal after propagation of various waves to the body surface.

(2) Relative timing of various EGM signals is very important as it could be employed to discriminate various cardiac events (e.g., SVT versus VT) accurately. For ECG signals, however, such timing information is not available.

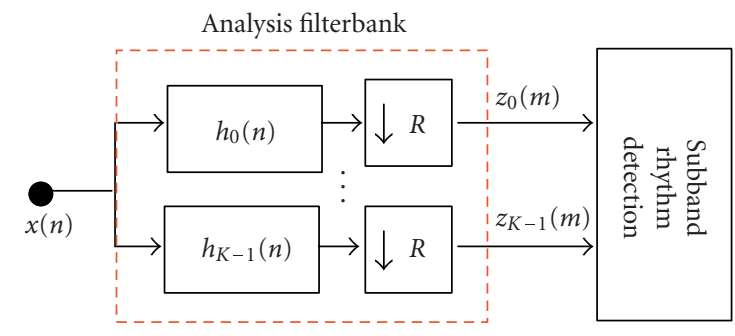

FIgURE 1: WOLA oversampled filterbank analysis for subband rhythm detection.

(3) Unlike ECG signals, sensed EGM signals are prone to cross-talk. For example, far-field R-waves (FFRWs) might occur when the much stronger ventricular signal interferes with sensing of the weaker atrial signal. Although FFRWs are a major problem with unipolar electrodes, they interfere with bipolar electrodes to a lesser degree.

Signal processing strategies are therefore greatly different for EGM signals as compared to ECG signals.

Rather than critically sampled polyphase filterbanks of [8], we employ a very efficient WOLA oversampled filterbank [6] for time-frequency analysis of the EGM signals. Subband peaks are detected directly from the subband signals (absolute values) by recursive averaging with no absolute thresholds. Subband peaks are combined in parallel by exploiting the synchrony of the subband signals at the beat time. Furthermore, to cope with the wide range of possible beat rates and morphologies of the EGM signal, the narrowband (complex) subbands are merged to obtain wideband-subband signals to be used for wideband event detection. The results of the wideband and narrowband detections are then combined for robust detection.

As the intention here is to describe the basis for the detection method, we limit our attention to single-electrode analysis; extension to multiple-electrode analysis is straightforward. Also, the algorithm simplicity has been a major consideration in this research since we are targeting low-power, real-time, and implantable applications.

This paper is organized as follows: Section 2 presents details of the detection algorithm, Section 3 discusses evaluation of the methods using the EGM signals in clean and in additive noise, and Section 4 presents research conclusions.

\section{THE PROPOSED DETECTION METHOD}

\subsection{General}

A time-domain EGM signal $x(n)$ is analyzed by an oversampled filterbank (depicted in Figure 1) that is efficiently implemented using a WOLA structure [6]. The filterbank parameters, adjusted by optimization for this application, are $K=32$ subbands, analysis window length of $L=256$, subband decimation factor of $R=4$, and oversampling factor of $\mathrm{OS}=K / R=8$. 
At the output of WOLA analysis, $K$ complex-valued subband signals are obtained: $Z_{k}(m), k=0,1, \ldots, K-1$, where $m$ is the subband time index. For real input signals, only half of the subbands are stored and processed due to Hermitian symmetry. The subband time-index $m$ is updated every $R=4$ input samples when a new block of WOLA subband signals is available.

Subband signals are then framed with a frame length of 3 seconds and a frame shift of 2 seconds. The frame length should be chosen long enough to cover more than one beat for slow beats (around 60 beats per minute, bpm) and to provide enough beats for statistical analysis. At the same time, the frame should be as short as possible to track the dynamics of the quickly varying beats. The choice of frame shift is rather arbitrary and depends on how often a decision is needed. Notice that irrespective of the frame length and frame shift, the WOLA analysis is continuously applied to the input signal, yielding a new block of subband signals for every $R$ input samples.

The cardiac beat is often represented by a sharp pulse in the EGM signal. As a result, the magnitudes of subband signals $\left(\left|Z_{k}(m)\right|, k=0,1, \ldots, K-1\right)$ exhibit mainly coherent peaks at the time of cardiac depolarization. A major objective in this research is to exploit this subband coherence (termed "synchrony" here) between various subbands. Among many possible methods, we designed a simple and robust approach based on binary operations to measure the synchrony.

Based on the synchrony analysis, a final beat sequence (called "optimal beat" in this paper) is detected for every frame as detailed in the next section. Then the periodicity and the regularity of the optimal beat are combined with the synchrony measure to detect the underlying cardiac event.

\subsection{Subband peak detection and synchrony analysis}

In the first stage, peaks are detected in selected subbands. Given the subband magnitude signal $\left|Z_{k}(m)\right|$ for subband $k$, its maximum is tracked with a two-time-constant firstorder recursive filter. Considering two filter coefficients of $0.9<\alpha_{m 1}<1$ and $0.1<\alpha_{m 2}<0.5$, the following pseudocode describes how the maximum signal $\left(M_{k}(m)\right)$ is calculated:

$$
\begin{gathered}
\alpha=\alpha_{m 1}, \\
\text { If }\left|Z_{k}(m)\right|>M_{k}(m-1), \quad \alpha=\alpha_{m 2}, \\
M_{k}(m)=M_{k}(m-1) \cdot \alpha+\left|Z_{k}(m)\right| \cdot(1-\alpha) .
\end{gathered}
$$

After each peak, the filter acts as a leaky integrator. Accordingly, the first filter coefficient $\left(\alpha_{m 1}\right)$ is selected close to one. This controls the so-called "release time" of the filter. The other coefficient is selected smaller for the filter to react quickly to the next peak.

Similarly, the average signal $A_{k}(m)$ is tracked by a twotime-constant recursive filter with $0.7<\alpha_{a 1}<1$ and $0.5<$ $\alpha_{a 2}<0.7$. At each time-instance $m$, a peak value $\left(P_{k}(m)\right)$ is detected by comparing the three values of $\left|Z_{k}(m)\right|, M_{k}(m)$,

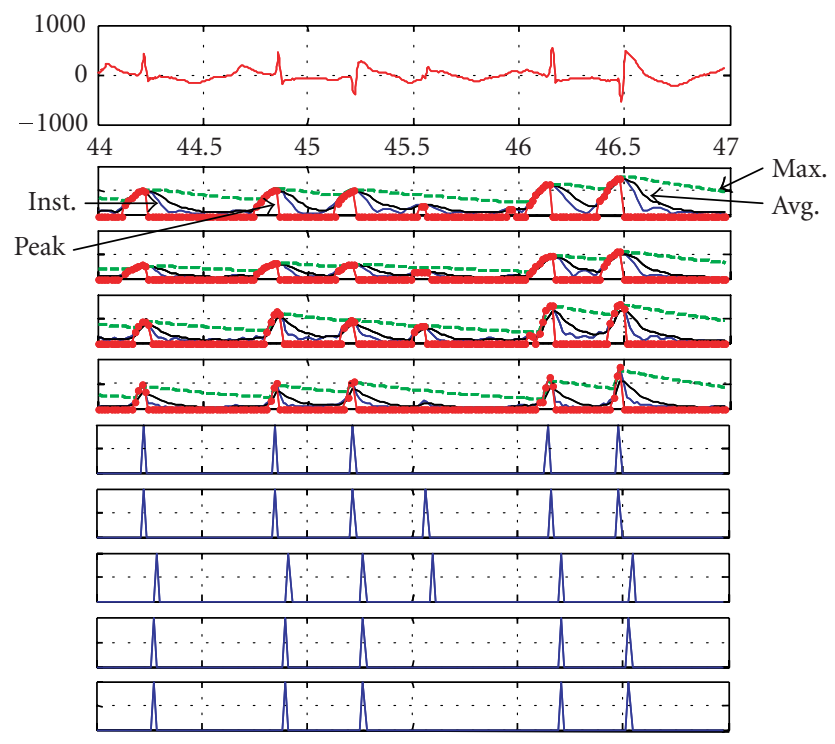

FIGURE 2: From top to bottom, a segment of ventricular EGM signal, four WOLA subband energies with their average, maximum, instantaneous, and peak signals, their corresponding binary pulses $B_{k}(m)$ (rows 6-9), and the optimal-detected pulse (bottom row).

and $A_{k}(m)$ (the instantaneous, maximum, and average values) as described in the following pseudocode:

$$
\begin{gathered}
P_{k}(m)=0, \\
\text { If }\left\{\left|Z_{k}(m)\right|>A_{k}(m-1) \&\left|Z_{k}(m)\right|>0.5 M_{k}(m-1)\right\} \& \\
\left\{A_{k}(m-1)<0.9 M_{k}(m-1)\right\}, \quad P_{k}(m)=\left|Z_{k}(m)\right| .
\end{gathered}
$$

Between two distant cardiac beats, it is possible that $A_{k}(m)$ and $M_{k}(m)$ converge to each other. To prevent peak detection in this situation, the last term in the condition above is included. Notice that no absolute threshold is used in the peak detection and only relative thresholds are employed.

By analyzing various beats in subband domain, it was observed that peaks appear more distinctively in the first half of the subbands. As a result, we limited peak detection to subbands 2-9 (out of 1-16). The first subband is ignored as it mostly captures noise and baseline wander. Figure 2 illustrates a frame of atrial EGM signal (top graph), and four sets of WOLA subband signals of $\left|Z_{k}(m)\right|, M_{k}(m), A_{k}(m)$, and $P_{k}(m)$ (instantaneous, maximum, average, and peak).

Following the peak detection, each subband peak signal $P_{k}(m)$ is converted to a binary $(0 / 1$ for peak/no-peak) signal $B_{k}(m)$. This greatly simplifies further processing. To embed more robustness in the algorithm and to avoid detecting short-term spurious peaks, we search every frame of binary peak signal $B_{k}(m)$ for a pattern of consecutive peaks (1s) followed by a block of zeros (e.g., $\left.\left\{\begin{array}{llllll}1 & 1 & 1 & 0 & 0 & 0\end{array}\right\}\right)$. For every pattern found, the falling edge of the binary peak signal is registered as a valid peak. The peak location is marked by a block of three 1 s $\left.\left(\begin{array}{lll}1 & 1 & 1\end{array}\right\}\right)$ and the rest of the peak signal is reset to zero. Replacing the peak by a block of 1 s (rather than a 
single 1) increases robustness in the next stage of synchrony analysis. All further steps of processing are applied to the binary peak signals $B_{k}(m)$.

\subsection{Synchrony analysis and robust beat detection}

In the next stage, the degree of synchrony between various subbands is measured by applying simple AND operations to the binary peak signals. For each possible pair of signals $B_{k}(m)$ and $B_{l}(m), k \neq l$, synchrony of the pair $S_{k, l}$ (in percentage) is calculated as follows:

$$
S_{k, l}=\frac{100 \mathrm{NP}\left(B_{k}(m) \& B_{l}(m)\right)}{\max \left\{\operatorname{NP}\left(B_{k}(m)\right), \operatorname{NP}\left(B_{l}(m)\right)\right\}},
$$

where function $\mathrm{NP}(\cdot)$ denotes the number of peaks in a frame of binary peaks and \& denotes the logical AND operation. The synchrony is evaluated for all nonidentical pairs (for 8 subbands this involves 28 AND operations on frame pairs). To minimize the effect of noise and interference, only the top 3 synchrony scores are considered as measures of the frame synchrony. The top 3 scores are compared to fixed synchrony thresholds to classify the frame of subband beats as perfectly synchronous ( $\mathrm{Syn}=4$ ), as borderline synchronous $($ Syn $=2)$, or asynchronous $($ Syn $=0)$.

We also employ the top 3 binary pulse pairs to robustly detect the beat times. Applying a majority-voting rule, beats are detected from the 3 pairs (after the logical AND operation within each pair) when 2 out of the 3 pairs exhibit simultaneous beats. Considering the peak extension to a block of three $1 \mathrm{~s}$, this method proved to be very robust when the signal quality was compromised by noise or due to flutter and fibrillation. As a result of beat detection, an optimal beat sequence $\mathrm{OB}(m)$ is obtained for every frame. Depicted in Figure 2 (rows 6-9) are binary subband pulses for the EGM segment together with the optimal-detected beat (bottom row).

\subsection{Analysis of periodicity and regularity}

Once an optimal beat sequence $\mathrm{OB}(m)$ is obtained, it is analyzed to find the beat rate and the regularity of the beats. A set of thresholds for periods of various cardiac events is used to set histogram edges as [0, FibPer/2, FibPer, FlutPer +1 , TachyPer +1 , SRMax, infinity]; where FibPer, FlutPer, TachyPer, and SRMax indicate the largest acceptable periods for fibrillation, flutter, tachycardia, and sinus rhythm, respectively. The beats are classified in a period histogram with 6 bins specified by the above edges. The mode (bin index for the most populated bin) of the histogram $\left(T_{m}\right)$ is an indicator of the periodicity. For the periods in the "acceptable" range of (FibPer/2, SRMax), the mean period $(\bar{T})$ and the standard deviation-to-mean ratio $(\sigma / \mu)$ are calculated. If either of the $\bar{T}$ and $T_{m}$ fall in the fibrillation, flutter or tachycardia range, the period zone indicator would be set to show the corresponding event. For sinus rhythm, however, both $\bar{T}$ and $T_{m}$ should indicate a sinus rhythm. In all cases, we chose to use the mean period $\bar{T}$ to find the rate as beat per minute, $\mathrm{bpm}=60 / \bar{T}$. So, it is possible that the period zone indicator show a flutter since $T_{m}$ is pointing to a flutter while average beat rate is still slightly below the minimum flutter rate.

$\sigma / \mu$ (of periods) is an indicator of the regularity; typically for very regular beats $\sigma / \mu<20 \%$, for very irregular beats $\sigma / \mu>40 \%$, and $\sigma / \mu$ values between the two ranges indicate moderate regularity. In case unusual lack of EGM activity (longer than the slowest possible rhythm) is detected within the frame, the irregularity flag is set (Ireg =1).

\subsection{Event detection based on subband features}

The synchrony analysis provides both the optimal beat $\mathrm{OB}(m)$ and the synchrony score (Syn $=0,2,4)$. Based on these and the periodicity and regularity of the optimal beat ( $\bar{T}, T_{m}, \sigma / \mu$, and Ireg), cardiac events are classified as one of the following eight events:

(1) stable sinus rhythm (SR),

(2) transitional SR (T-SR),

(3) stable tachycardia (VT or AT),

(4) transitional tachycardia (T-VT or T-AT),

(5) flutter (VFLUT or AFLUT),

(6) fibrillation (VFIB or AFIB),

(7) synchronous but irregular rhythm (Syn-Irg),

(8) unclassified,

where VT, VFLUT, and VFIB represent ventricular events of tachycardia, flutter, and fibrillation, respectively. Similarly, AT, AFLUT, and AFIB represent the corresponding atrial events. When the mean period is within the range for sinus rhythm but the rhythm is irregular, a transitional event of T-SR is detected. A similar criterion is used in detection of TVT or T-AT. Event (7) is detected when the synchrony is perfect but periods are too irregular or insufficient in number to be considered for other classes. Finally, event (8) is reserved for unclassified rhythms.

A flowchart of the event detection algorithm is depicted in Figure 3. As shown, the algorithm sets a series of traps for various events. It first tries to identify fibrillation or flutter (classes $\{5,6\}$ ). If none is detected (state $\mathrm{A}$ in the figure), it searches for fast beats (classes $\{3,4,7\}$ ) and then sinus rhythm (classes $\{1,2,7\}$ ). If none of the traps succeeds in detection, the beat remains unclassified (class 8).

\subsection{Detection by wideband filterbank}

The expected range of cardiac beat rates is very wide, from less than $50 \mathrm{bpm}$ to over $300 \mathrm{bpm}$. As a result of the classic time-frequency resolution trade-off, the time resolution of the narrowband filterbank (WOLA analysis with $K=32$ ) is insufficient to separate two closely spaced beats. The problem is compounded when the signal quality is further compromised during flutter or fibrillation.

An effective solution is to use a filterbank with wider subbands. In uniform filterbanks, it is possible to merge the subbands through a simple postprocessing [9]. Specifically, in the WOLA filterbank, we can combine, for example, every neighboring pair of complex subband signals to obtain a wideband analysis, doubling the time resolution. Since all 

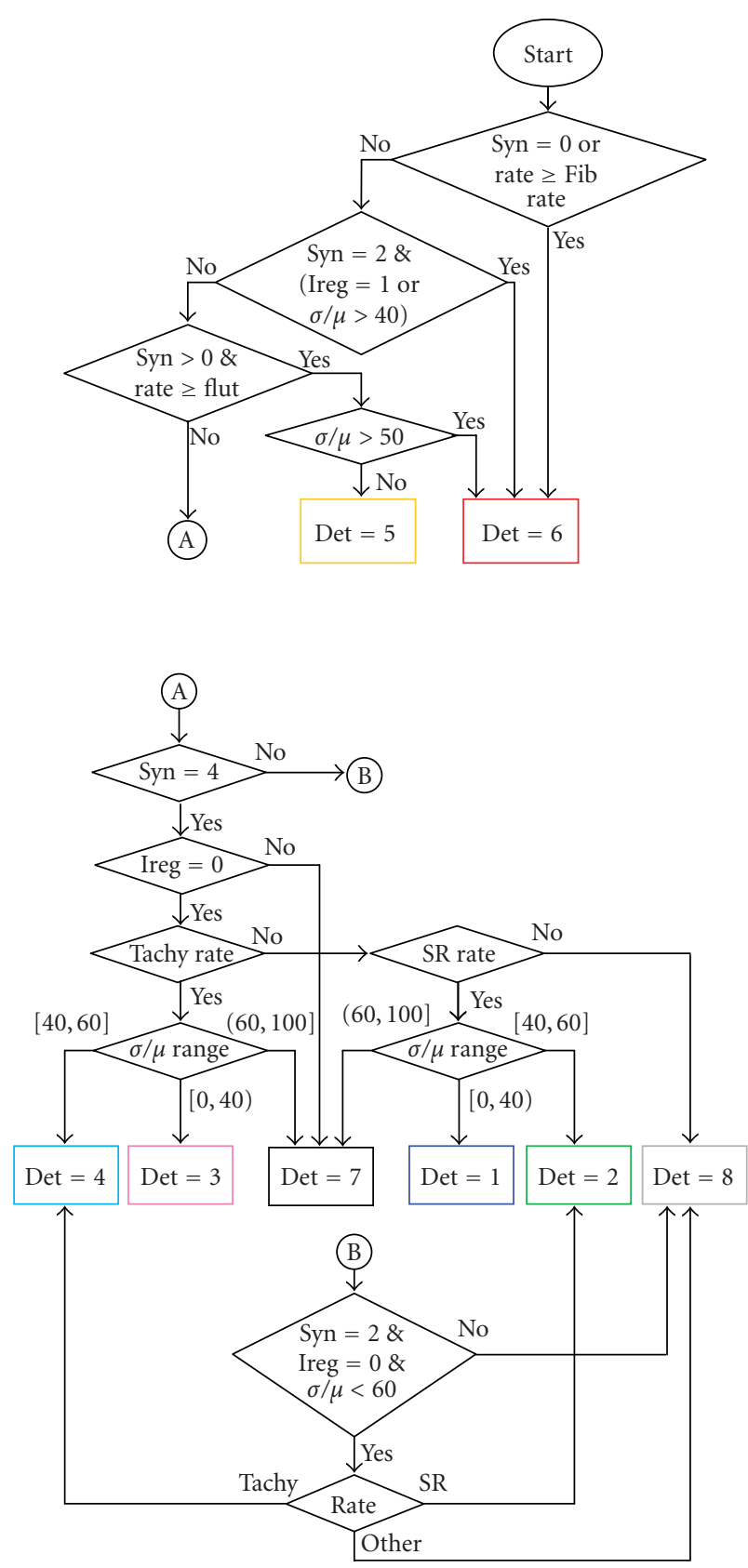

FIgURE 3: Flowchart of the event detection algorithm.

of the subband signals have baseband spectrums, to combine subbands one has to modulate the bands to line them up sequentially. For merging two subbands, for example, one has to apply a complex modulation to the higher-frequency subband and add the results with the lower-frequency one.

To achieve a higher temporal resolution, we combined the low-frequency subbands in pairs (subbands 2-7) and in a group of four (subbands 2-5) resulting in four new wider subband signals. In merging subbands, the limiting factor is the filterbank oversampling factor $(\mathrm{OS}=K / R)$. As the effective number of bands $(K)$ decreases for wider subbands, the potential for aliasing increases. The aliasing is kept minimal with our proposed WOLA setup $(K=32$, OS $=8$ ), when grouping in pairs (equivalent to $K=16$ bands) or in fours (effective $K$ of 8 ) since the oversampling factor for the combined bands is at least $O S=2$. Aliasing and distortion are also greatly reduced by proper prototype filter design but we refrain from discussion here for brevity.

Using the four wider subbands, we applied a wideband peak detection, synchrony, periodicity, and regularity analysis similar to the narrowband case.

\subsection{Low-frequency detection}

The EGM wave morphology is very diverse. Among all various forms, there are cases where the EGM beat lacks a clear strong impulse at the beat instance. Instead, a periodic waveform with wide R-waves or P-waves (for the ventricular or atrial signals, resp.) is observed with weak impulses at the beat locations. Detecting such beats is problematic in noise since the EGM waveform, exhibiting a low-pass behavior, is presented mostly in very low frequency bands. To increase noise robustness for such cases, we added a third method of beat detection by using only the peaks detected in subbands 2 and 3. The synchrony between the two subbands as well as the periodicity and regularity of optimal beat (AND result of the two) is calculated as before. This is called low-frequency (LF) detection here.

\subsection{Multitiered beat and event detection}

Taking the narrowband filterbank detection as the default, the wideband system is selected when all of the following conditions are met.

(1) Wideband detection shows perfect synchrony (Syn=4).

(2) Wideband detection has $\sigma / \mu<40 \%$ or less than the corresponding value for the narrowband detection.

Switching to the LF detection occurs when all of the following conditions are met.

(1) Both the narrow and wideband systems are not synchronous (Syn $<4$ ); or $\sigma / \mu$ of the LF detection is superior to (less than) each of the other two systems by at least $40 \%$.

(2) The LF system detects less than four pulses in the frame.

(3) The LF system is not detecting fibrillation or flutter.

\section{SYSTEM PERFORMANCE EVALUATION}

The EGM data from Vol. I of the Ann Arbor Electrogram Libraries (AAEL) [10] was used for system evaluation. For most experiments, the EGM signals recorded with bipolar electrodes were utilized since they provide the best quality. The two bipolar signals were recorded from Right Ventricular Apex and High Right Atrium, called RVAb and HRAb in the AAEL documentation, respectively. All of the RVAb and $\mathrm{HRAb}$ signals (more than 330 minutes of EGM data for 60 patients in 214 files) were used for system evaluation. 

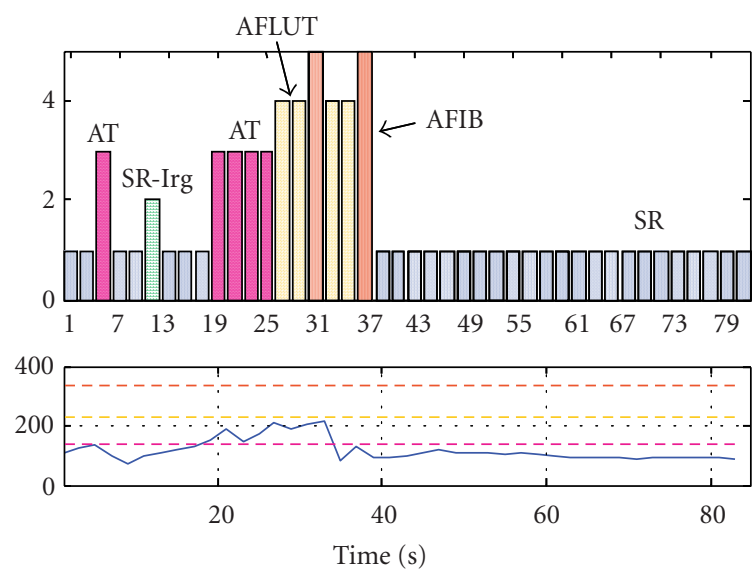

FIGURE 4: Algorithm performance for an HRAb EGM signal (AAEL file: SET1, A241563.SIG), top view: detected events in time, bottom view: detected rate in beats per minute (solid line) in time, and AFIB, AFLUT, and AT rate thresholds (dashed lines, from top to bottom, resp.).

To evaluate the system performance for FFRWs, we also employed most of the usable High Right Atrium unipolar (HRAu) signals from the database.

The EGM signal was digitally decimated from the original sampling frequency of $1000 \mathrm{~Hz}$ down to Fs $=250 \mathrm{~Hz}$. Using a small subset of the AAEL representing various events, the system parameters were tuned until there were no missed events, and the frame-by-frame classification was as accurate as possible. Then, for every case in the AAEL library, the detection algorithm was tested and the results were compared to the physician-certified AAEL annotations. Figure 4 depicts a typical output summary of the detection system showing various detected events and the beat rate. Notice that there is no "training" involved in the proposed detection system; rather system parameters had to be optimized on a subset of the EGM data.

\subsection{Statistical performance evaluation}

After optimizing the detection system and testing on the whole database in clean, we needed to evaluate the performance of the system in noise. Also of interest was the performance of reduced versions of the detection algorithm in clean and in noise. For practical applications, noise robustness is a very desired feature of any cardiac event detection. For a statistical analysis, we compared the performance in noise (or with reduced algorithms) against the benchmark detection, that is, the results for the algorithm in clean. Frames representing cardioversion, lead failure, and lead dislodgement (in total around 28 minutes) were excluded from the comparisons.

To simulate various noise conditions, five different noises were added to the EGM signals:

(1) white Gaussian noise, $(0, \pi)$ band,

(2) lowpass noise, $(0, \pi / 4)$ band,
(3) bandpass noise, $(\pi / 4, \pi / 2)$ band,

(4) highpass noise, $(3 \pi / 4, \pi)$ band,

(5) tonal $60 \mathrm{~Hz}$ noise.

Noises (2)-(4) were obtained by filtering white noise. To adjust the noise level for a given signal-to-noise ratio (SNR), one needs to measure the EGM signal power. Due to the variability of the EGM signal in terms of magnitude, polarity, and morphology (very wide waves to very sharp ones), measurement of long-term power is inadequate. Instead, we adjusted the noise level based on tracking the short-term (4 second) EGM signal envelope (rather than power).

To evaluate the performance for EGM signal corrupted with additive noise, output summaries (similar to that of Figure 4) were carefully compared to the output summaries for the clean EGM signals to ensure that no block of event was lost. This was done for the whole EGM database (214 signal files). Increasing the noise level, the detection system performed well up to $15 \mathrm{~dB} S N R$ for noise types 1-3, while for noises 4-5 the performance was fine up to $0 \mathrm{~dB} S N R$ noise power.

To quantify the performance, we grouped the 8 cardiac events (Section 2.5) in two separate groups, $\{1-4\}$ (SR/Tachy) and \{5-6\} (Fib/Flut), and measured the detection performance using frame counts of TP, TN, FP, and FN defined as follows.

(1) TP, true positive: correct detection of $\{5-6\}$.

(2) TP, true negative: correctly not detecting $\{5,6\}$.

(3) FP, false positive: falsely detecting $\{5,6\}$.

(4) FN, false negative: falsely not detecting $\{5,6\}$.

From these frame counts, the Fib/Flut positive predictivity $(+P)$ and negative predictivity $(-P)$ were calculated as follows:

$$
+P=\frac{\mathrm{TP}}{\mathrm{TP}+\mathrm{FP}}, \quad-P=\frac{\mathrm{TN}}{\mathrm{TN}+\mathrm{FN}} .
$$

Depicted in Table 1 are the total frame counts of TP, FP, TN, and FN for the five noise types. Also, Table 2 summarizes the $+P$ and $-P$ measures for the five noises. As expected, the adverse effects of white noise on subband detection are worse as they corrupt all the bands equally. On the other extreme, the system shows immunity to even $0 \mathrm{~dB}$ SNR tonal $(60 \mathrm{~Hz})$ and highpass noises. Our careful observations revealed that no block event was missed or misrecognized with five noise types in Table 2. Moreover, most of the recognition errors in noise occurred before or after fibrillation or flutter events when the quality of EGM signal was already compromised.

\subsection{Evaluation in presence of FFRWs}

As described in Section 1, FFRWs pose more difficulties when sensing with unipolar electrodes. We analyze the system performance in the presence of FFRWs to demonstrate the general robustness of the detection system to interferences.

In the AAEL Vol. I EGM database, FFRWs are present in a few cases (encompassing 19 files in sets 1 and 8) that include usable (noise-free sensing) unipolar recording of the 
TABLE 1: Number of TP, FP, TN, and FN frames for five noise types of (1) white, (2) lowpass, (3) bandpass, (4) highpass, and (5) $60 \mathrm{~Hz}$, with noise types (1)-(3) at $15 \mathrm{~dB}$ and types (4)-(5) at $0 \mathrm{~dB}$ SNR.

\begin{tabular}{|c|c|c|c|c|c|}
\hline & & \multicolumn{4}{|c|}{ Actual } \\
\hline & & \multicolumn{2}{|c|}{ FIB/FLUT } & \multicolumn{2}{|c|}{ SR/VT } \\
\hline \multirow{12}{*}{ 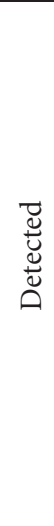 } & \multirow{6}{*}{ FIB/FLUT } & \multicolumn{2}{|c|}{ True positive } & \multicolumn{2}{|c|}{ False positive } \\
\hline & & (1) & 1005 & (1) & 43 \\
\hline & & (2) & 1114 & (2) & 27 \\
\hline & & (3) & 1001 & (3) & 11 \\
\hline & & (4) & 1027 & (4) & 0 \\
\hline & & (5) & 1020 & (4) & 0 \\
\hline & \multirow{6}{*}{ SR/VT } & \multicolumn{2}{|c|}{ False negative } & \multicolumn{2}{|c|}{ True negative } \\
\hline & & (1) & 38 & (1) & 8777 \\
\hline & & (2) & 29 & (2) & 8786 \\
\hline & & (3) & 42 & (3) & 8825 \\
\hline & & (4) & 16 & (4) & 8895 \\
\hline & & (5) & 23 & (5) & 8882 \\
\hline
\end{tabular}

TABLE 2: Positive and negative predictivity for five noise types of (1) white, (2) lowpass, (3) bandpass, (4) highpass, and (5) $60 \mathrm{~Hz}$.

\begin{tabular}{lc|r|c}
\hline \multicolumn{2}{c|}{ Noise type } & $+P \%$ & $-P \%$ \\
\hline$(1)$ & 15 dB SNR & 95.9 & 99.6 \\
$(2)$ & 15 dB SNR & 97.4 & 99.7 \\
$(3)$ & 15 dB SNR & 98.9 & 99.5 \\
$(4)$ & 0 dB SNR & 100.0 & 99.8 \\
$(5)$ & 0 dB SNR & 100.0 & 99.7 \\
\hline
\end{tabular}

atrial signal (HRAu signal). As the ventricular interfering signal propagates through the heart medium to reach the atrial electrode; it is inevitably filtered by the medium. As a result, the subband synchrony of the interfering beats is much weaker than the synchrony of the atrial signal, even when the FFRWs are larger in magnitude. This enables the subbandbased detection system to reject the FFRWs safely. Figure 5 depicts a segment of the EGM signal sensed by a unipolar electrode (HRAu signal) including clear FFRW interference. For comparison, the same segment sensed by the bipolar electrode (HRAb signal) is also shown. The detected beats (c) are the same for both HRAb and HRAu signals demonstrating the robustness of the detection to FFRWs. We employed all available FFRW cases (19 files) for evaluation by comparing the results with the HRAb signal. The detection system performed reliably in 16 (out of 19) cases. In 3 cases, the FFRWs were so dominant that the detection system could not discriminate them from the atrial beats.

\section{CONCLUSIONS}

The subband-based methods proposed in this paper for processing intracardiac EGM signals offer a robust and reliable performance by employing parallel narrowband peak detectors. Proper and efficient combination of subband peaks by synchrony analysis is a major milestone in this research.

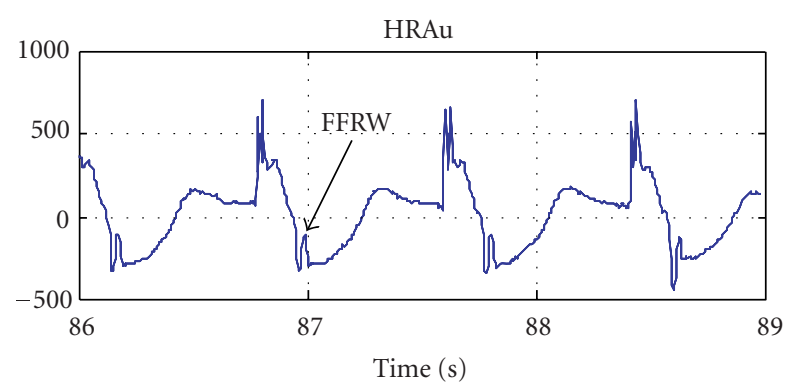

(a)

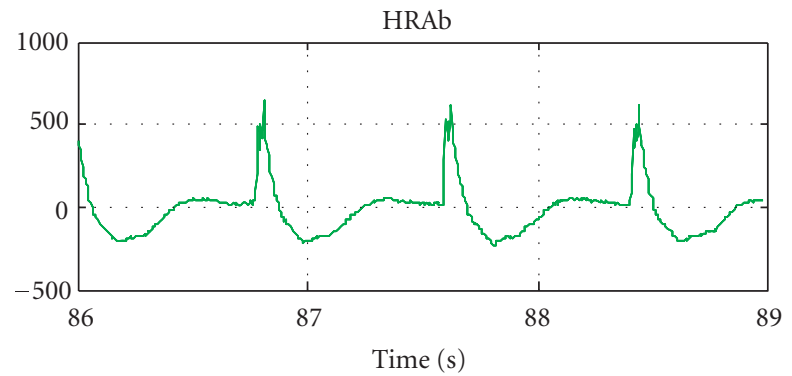

(b)

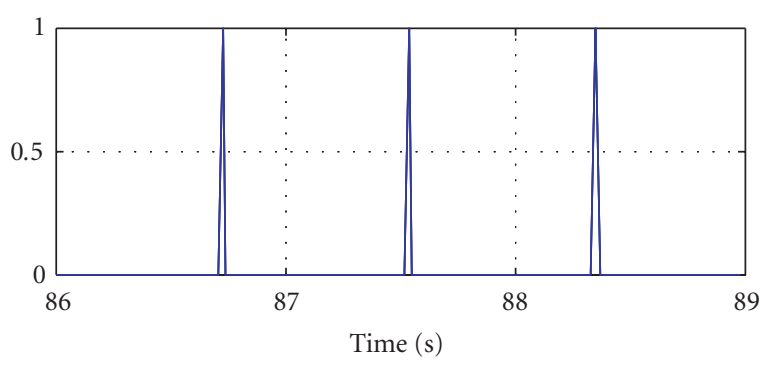

(c)

FIGURE 5: The EGM signals of (a) HRAu (with FFRW interference), (b) HRAb, and (c) the detected beats.

The method, extensively evaluated using the AAEL EGM database, demonstrates excellent performance in terms of accurate event detection and beat-rate measurement even in fibrillation or flutter when the signal quality is compromised. Evaluation in noise and in the presence of far-field R-waves has also demonstrated significant robustness to noise and interference. This method is simple enough for implementation on an ultra-low power WOLA filterbank platform and requires only simple operations as a result of using binary peak signals.

For future work, we propose the use of subband features for morphology analysis and pattern matching. This could outperform comparable time-domain methods due to superior and more robust representation of signal spectral features in the subband domain. Also, research is being conducted towards an ultra-low power implementation of the WOLA-based detection algorithm at power levels comparable to current ICDs (typically less than one $\mu \mathrm{W}$ ). Our initial findings confirm the feasibility of this implementation. 
The proposed robust WOLA-based detection method may beneficially be combined with time-domain methods when time response is crucial (e.g., in pacing).

\section{REFERENCES}

[1] M. Astrom, S. Olmos, and L. Sornmo, "Wavelet-based event detection in implantable cardiac rhythm management devices," IEEE Transactions on Biomedical Engineering, vol. 53, no. 3, pp. 478-484, 2006.

[2] M. L. Brown, J. L. Christensen, and J. M. Gillberg, "Improved discrimination of VT from SVT in dual-chamber ICDs by combined analysis of dual-chamber intervals and ventricular electrogram morphology," in Proceedings of of the 29th Annual Meeting on Computers in Cardiology, pp. 117-120, Memphis, Tenn, USA, September 2002.

[3] L. A. Koyrakh, J. M. Gillberg, and N. M. Wood, "Wavelet transform based algorithms for EGM morphology discrimination for implantable ICDs," in Proceedings of the 26th Annual Meeting on Computers in Cardiology, pp. 343-346, Hannover, Germany, September 1999.

[4] J. L. Rojo-Alvarez, A. Arenal-Maiz, and A. Artes-Rodriguez, "Discriminating between supraventricular and ventricular tachycardias from EGM onset analysis," IEEE Engineering in Medicine and Biology Magazine, vol. 21, no. 1, pp. 16-26, 2002.

[5] B. Schaer and S. Osswald, "Methods of minimizing inappropriate implantable cardioverter-defibrillator shocks," Current Cardiology Reports, vol. 2, no. 4, pp. 346-352, 2000.

[6] R. Brennan and T. Schneider, "Flexible filterbank structure for extensive signal manipulations in digital hearing aids," in Proceedings of IEEE International Symposium on Circuits and Systems (ISCAS '98), vol. 6, pp. 569-572, Monterey, Calif, USA, May 1998.

[7] B.-U. Kohler, C. Hennig, and R. Orglmeister, "The principles of software QRS detection," IEEE Engineering in Medicine and Biology Magazine, vol. 21, no. 1, pp. 42-57, 2002.

[8] V. X. Afonso, W. J. Tompkins, T. Q. Nguyen, and S. Luo, "ECG beat detection using filter banks," IEEE Transactions on Biomedical Engineering, vol. 46, no. 2, pp. 192-202, 1999.

[9] R. L. de Queiroz, "Uniform filter banks with nonuniform bands: post-processing design," in Proceedings of IEEE International Conference on Acoustics, Speech and Signal Processing (ICASSP '98), vol. 3, pp. 1341-1344, Seattler, Wash, USA, May 1998.

[10] Ann Arbor Electrogram Libraries, Ann Arbor MI, USA, http:// electrogram.com/.

Hamid Sheikhzadeh obtained his B.S. (1986) and M.S. (1989) degrees in electrical engineering both from Amirkabir University of Technology (AUT) in Tehran. He received his Ph.D. degree from the E\&CE Department of the University of Waterloo in Canada in 1994 and continued his research as a Postdoctoral Fellow for about a year. From October 1994, he served as a Faculty Member of the EE Department of AUT

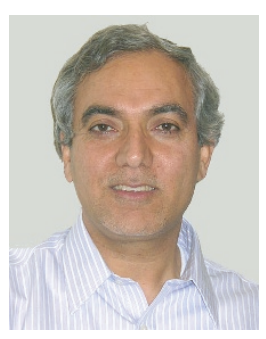
for six years. In the meantime, he held positions as Vice Chairman of Academic Affairs and Head of Communications Group of the EE Department for about two years each. In November 2000, he joined the early research team at AMI Semiconductor Canada (then Dspfactory Ltd.) and since then has been working as a Senior
Member of the R\&D team. Also, since April 2001, he has been collaborating with the E\&CE Department of University of Waterloo as an Adjunct Professor. His research interests include digital signal processing, speech and audio processing, modeling of the central and peripheral auditory system, adaptive signal processing, signal processing for ultra-low power and portable devices, and biomedical signal processing. He is a Senior Member of IEEE and a Member of ISCA.

Robert L. Brennan received the B.A.Sc., M.A.Sc. degrees in electrical engineering from the University of Waterloo in Canada in 1985 and 1986, respectively. He received his Ph.D. degree in 1991 from the University of Waterloo, investigating algorithms and architectures for extremely low bitrate speech coders. At Unitron, a Canadian manufacturer of hearing aids, he led the company toward developing high perfor-

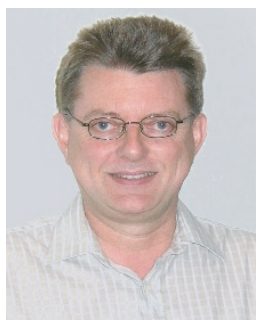
mance digital architectures replacing the almost exclusive analog technology deployed at the time. This work has expanded and continued through the formation of Dspfactory and now, AMI Semiconductor. As a Senior Scientist, he continues working on digital signal processing and filterbank multirate methods in audio, industrial, automotive, and medical applications.

Simon So is in his final year of the undergraduate program at the Systems Design Engineering Department of the University of Waterloo. In 2005, during his work term at AMI Semiconductor Canada Company in Waterloo, he worked as a Member of the research and development team on Cardiac Rhythm Management project, contributing to the current paper. His current interests include digital signal processing, digital video and image processing, and video/audio compression algorithms.

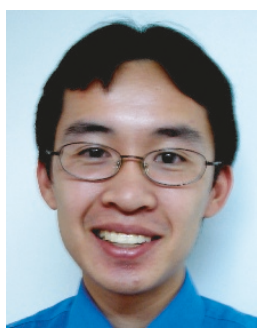

